\title{
Plasma Levels of Pentosidine, Carboxymethyl-Lysine, Soluble Receptor for Advanced Glycation End Products, and Metabolic Syndrome: The Metformin Effect
}

\author{
Mohamed Haddad, ${ }^{1}$ Ines Knani, ${ }^{1}$ Hsan Bouzidi, ${ }^{2}$ Olfa Berriche, ${ }^{3}$ \\ Mohamed Hammami, ${ }^{1}$ and Mohsen Kerkeni ${ }^{1}$ \\ ${ }^{1}$ Laboratory of Biochemistry (LR12ES05) "Nutrition-Aliment Fonctionnel et Santé vasculaire", Faculty of Medicine, \\ University of Monastir, Monastir, Tunisia \\ ${ }^{2}$ Laboratory of Biochemistry, CHU Thar Sfar, Mahdia, Tunisia \\ ${ }^{3}$ Laboratory of Endocrinology, CHU Thar Sfar, Mahdia, Tunisia
}

Correspondence should be addressed to Mohsen Kerkeni; mohsen.kerkeni@yahoo.fr

Received 21 June 2016; Revised 27 August 2016; Accepted 19 September 2016

Academic Editor: Eugene H. J. M. Jansen

Copyright (c) 2016 Mohamed Haddad et al. This is an open access article distributed under the Creative Commons Attribution License, which permits unrestricted use, distribution, and reproduction in any medium, provided the original work is properly cited.

\begin{abstract}
Metabolic syndrome (MetS) is considered one of the most important public health problems. Several and controversial studies showed that the role of advanced glycation end products (AGEs) and their receptor in the development of metabolic syndrome and therapeutic pathways is still unsolved. We have investigated whether plasma pentosidine, carboxymethyl-lysine (CML), and soluble receptor for advanced glycation end products (sRAGE) levels were increased in patients with MetS and the effect of metformin in plasma levels of pentosidine, CML, and sRAGE. 80 control subjects and 86 patients were included in this study. Pentosidine, CML, and sRAGE were measured in plasma by enzyme-linked immunosorbent assay (ELISA). Plasma pentosidine, CML, and sRAGE levels were significantly increased in patients compared to control subjects $(P<0.001, P<0.001$, and $P=0.014$, resp.). Plasma levels of pentosidine were significantly decreased in patients who received metformin compared to untreated patients $(P=0.01)$. However, there was no significant difference between patients treated with metformin and untreated patients in plasma CML levels. Plasma levels of sRAGE were significantly increased in patients who received metformin and ACE inhibitors $(P<0.001$ and $P=$ 0.002 , resp.). However, in a multiple stepwise regression analysis, pentosidine, sRAGE, and drugs treatments were not independently associated. Patients with metabolic syndrome showed increased levels of AGEs such as pentosidine and CML. Metformin treatment showed a decreased level of pentosidine but not of CML. Therapeutic pathways of AGEs development should be taken into account and further experimental and in vitro studies merit for advanced research.
\end{abstract}

\section{Introduction}

Cardiovascular diseases are one of the main causes of mortality in industrialized countries, due to an increase in prevalence of different cardiovascular risk such as abdominal obesity, insulin resistance, dyslipidemia, hypertension, and hyperglycemia. These metabolic disorders presented within the same individual give rise to metabolic syndrome. Recent estimates are that between 20 and $30 \%$ of the adult population worldwide have a metabolic syndrome [1]. The causes of this disease are multifactorial, stemming from complex genetic and environmental influences such as physical inactivity, smoking, and a diet rich in sugars and saturated fatty acids.

Due to complex metabolic disorder in metabolic syndrome, reactive derivatives are formed via nonenzymatic reaction named Maillard reaction between the free amino groups in lysine and arginine residues in proteins and carbohydrates that undergo a series of complex reactions to an irreversible complex group of compound termed advanced glycation end products (AGEs) $[2,3]$. These products can be formed by covalent binding of methylglyoxal (MG) and glyoxal to the free amino and thiol groups of proteins [4]. 
In addition to their endogenous formation, AGEs exist in high amounts in cooked fast-food diet and known by brown coloration, but only a small quantity of these compounds present in food are absorbed and the other part is secreted by the kidneys in urine [5]. However, pentosidine and $\varepsilon-\mathrm{N}$ carboxymethyl-l-lysine (CML) have been well characterized as biomarkers for the formation and accumulation of AGEs [6] and are known to play an important role in diabetes and vascular complications [7-9]. AGEs cause damage by affecting protein structure, by formation of cross-links between molecules, or by binding the receptor for AGEs (RAGE) [1012]. RAGE is a member of the immunoglobulin superfamily of cell surface proteins that binds AGEs and other molecules.

Previous studies have confirmed that interaction between AGEs and RAGE activates the generation of oxidative stress and production of proinflammatory cytokines in various types of cells that have been implicated in metabolic and many other disease statuses [13-16]. Furthermore, Monden et al. have shown that RAGE affects adipocyte hypertrophy and insulin sensitivity in mice [17]. In addition to the membrane form, RAGE is found in the soluble form (sRAGE) and two different types are identified. sRAGE formed by the cleavage of RAGE by metalloproteinases ADAM10 and MMP9 [18]. esRAGE (endogenous secretory RAGE) formed by the alternative splicing of RAGE mRNA. Because of its ability to bind with the same ligands, sRAGE acts as a competitive inhibitor of RAGE and precludes the cell-bound RAGE signaling [19]. For this reason, many clinical studies have concluded the possibility of using sRAGE as a disease marker in such disorders [20,21]. Several and controversial studies showed that the roles of AGEs and their receptor in the development of metabolic syndrome and therapeutic pathways to decrease the level of these biomarkers are still unsolved.

The present study was designed to elucidate, in the first part, the level of serum pentosidine, CML, and soluble form of RAGE (sRAGE) in patients with metabolic syndrome and, in the second part, to investigate the effect of several drugs, in particular, metformin which is known by its antiglycation effect, on pentosidine, CML, and sRAGE levels in these patients.

\section{Subjects and Methods}

2.1. Subjects. This study included 80 control subjects and 86 patients who developed a metabolic syndrome recruited from Department of Endocrinology of Taher Sfar Hospital in Mahdia (Tunisia). The criteria of the American Heart Association Scientific Statements of 2009 were used to define metabolic syndrome [22]. Patients were considered to have metabolic syndrome if they presented 3 or more of the following risk factors: (1) elevated waist circumference ( $\geq 90 \mathrm{~cm}$ for men and $\geq 80 \mathrm{~cm}$ for women); (2) elevated TG ( $\geq 1.7 \mathrm{mM}$ ) (drug treatment for elevated triglycerides is an alternate indicator); (3) reduced HDL-C ( $<1 \mathrm{mM}$ for men and $<1.3 \mathrm{mM}$ for women) (drug treatment for reduced HDL-C is an alternate indicator); (4) elevated blood pressure (systolic BP $(\mathrm{SBP}) \geq 130 \mathrm{mmHg}$ or diastolic BP (DBP) $\geq 85 \mathrm{mmHg}$ ) (antihypertensive drug treatment in a patient with a history of hypertension is an alternate indicator); and (5) elevated fasting glucose $(\geq 5.5 \mathrm{mM}$ ) (drug treatment of elevated glucose is an alternate indicator). Data collection was performed using an information sheet, specifically designed and containing all the personal information as well as the various elements of metabolic syndrome. For this, a clinical examination was carried out to determine the weight, height, waist circumference, blood pressure, and the drug treatments from each patient. In fact, patients were divided into patients who are treated with metformin (Glucophage $850 \mathrm{mg} ; 3$ times/day) $(n=55)$ and patients who are untreated with this drug $(n=31)$.

2.2. Methods. In all subjects, venous blood was collected in the morning after an overnight fast. After centrifuging the samples at $3000 \mathrm{rpm}$ for $10 \mathrm{~min}$ at $4^{\circ} \mathrm{C}$, plasma and serum were collected and stored at $-80^{\circ} \mathrm{C}$ until analysis. Fasting plasma glucose, triglyceride, total cholesterol, HDL cholesterol, and LDL cholesterol were measured using enzymatic methods by CX9 autochemical analysis instrument (Bechman CX9, USA). Hemoglobin A1C (HbAlc) was measured using G7 HPLC Analyser (Tosoh Europe NV). Plasma levels of pentosidine, CML, and sRAGE were determined by enzyme-linked immunosorbent assay (ELISA) provided by Cusabio Biotech Co., Ltd., according to the manufacturer's instructions. Detection range of pentosidine CML and sRAGE kit was, respectively, 31-2000 pmol/mL, $62-4000 \mathrm{pg} / \mathrm{mL}$, and $78-5000 \mathrm{pg} / \mathrm{mL}$. The intra-assay and interassay coefficients of variation were $<8 \%$ and $<10 \%$, respectively.

2.3. Statistical Analysis. The study data was expressed as mean \pm SD or median and interquartile ranges (IQR) using the SPSS program (version 18). Differences between groups were analyzed by the independent sample Student's $t$-test or Mann-Whitney $U$ test. A $P$ value less than 0.05 was considered statistically significant. Correlation was determined by a linear regression analysis and a multiple regression analysis was used to further explore the linear relationships between the variables. Regression variables were estimated as well as the correlation coefficient $r$. ANOVA was used to assess the significance of the regression with significance accepted at $P<0.05$.

\section{Result}

3.1. Clinical and Anthropometric Characteristics. The clinical, anthropometric characteristics and treatment drugs of controls and patients with MetS are shown in Table 1. No difference between the mean age of control subjects and patients was found. In total, $86 \%$ of patients were diabetic, $69.8 \%$ had hypertension, and $33.7 \%$ had hyperlipidaemia. Significant differences were seen between the groups in waist circumference, body mass index, serum glucose, HbAlc, triglyceride $(P<0.001)$, and total and HDL cholesterol $(P<$ $0.01)$.

3.2. Pentosidine, CML, and sRAGE Levels in Control Subjects and Patients with MetS. Table 2 shows results of plasma pentosidine, CML, and sRAGE levels in patients and 
TABLE 1: Clinical and anthropometric parameters of control subjects and patients with MetS.

\begin{tabular}{|c|c|c|c|}
\hline Characteristic & $\begin{array}{c}\text { Control subjects } \\
n=80\end{array}$ & $\begin{array}{c}\text { Patients } \\
n=86\end{array}$ & $P$ \\
\hline $\operatorname{Sex}(M / F)$ & $(41 / 39)$ & $(40 / 46)$ & NS \\
\hline Age (years) & $57 \pm 11$ & $59 \pm 12$ & NS \\
\hline Waist circumference $(\mathrm{cm})$ & $84.5 \pm 5.4$ & $109.8 \pm 13.4$ & $<0.001$ \\
\hline BMI $\left(\mathrm{kg} / \mathrm{m}^{2}\right)$ & $20.8 \pm 1.5$ & $33.3 \pm 8.6$ & $<0.001$ \\
\hline Diabetes, $n(\%)$ & $0(0)$ & $74(86)$ & - \\
\hline Hypertension, $n(\%)$ & $0(0)$ & $60(69.8)$ & - \\
\hline Dyslipidemia, $n(\%)$ & $0(0)$ & $29(33.7)$ & - \\
\hline Glucose $(\mathrm{mmol} / \mathrm{L})$ & $4.68 \pm 0.5$ & $12.8 \pm 6.5$ & $<0.001$ \\
\hline $\operatorname{HbAlc}(\%)$ & $5.1 \pm 0.31$ & $9.6 \pm 2.3$ & $<0.001$ \\
\hline Triglyceride (mmol/L) & $0.89(0.62-1.2)$ & $1.72(1.39-2.51)$ & $<0.001$ \\
\hline Total cholesterol (mmol/L) & $3.15(0.88-4.94)$ & $5(3.67-5.47)$ & $<0.01$ \\
\hline HDL cholesterol (mmol/L) & $1.2 \pm 0.2$ & $1.04 \pm 0.2$ & $<0.01$ \\
\hline LDL cholesterol (mmol/L) & $2.67(1.99-3.65)$ & $2.8(2.2-3.6)$ & NS \\
\hline $\mathrm{SBP}(\mathrm{mmHg})$ & 130 & $133.9 \pm 17$ & NS \\
\hline $\mathrm{DBP}(\mathrm{mmHg})$ & 80 & $72 \pm 8$ & $<0.001$ \\
\hline Lipid-lowering drugs, $n(\%)$ & $0(0)$ & $22(25.6)$ & - \\
\hline ACE inhibitors, $n(\%)$ & $0(0)$ & $33(38.4)$ & - \\
\hline ARBs, $n(\%)$ & $0(0)$ & $11(12.8)$ & - \\
\hline Diuretics, $n(\%)$ & $0(0)$ & $16(18.6)$ & - \\
\hline Calcium channel blockers, $n(\%)$ & $0(0)$ & $26(30.2)$ & - \\
\hline Insulin, $n(\%)$ & $0(0)$ & $38(44.2)$ & - \\
\hline Metformin, $n(\%)$ & $0(0)$ & $55(64)$ & - \\
\hline
\end{tabular}

Values are mean \pm standard deviation or median (25\%-75\%) percentiles; MetS: metabolic syndrome; BMI: body mass index; HDL: high density lipoprotein; LDL: low density lipoprotein; SBP: systolic blood pressure; DBP: diastolic blood pressure; ACE: angiotensin converting enzyme; ARBs: angiotensin receptor blockers; NS: not significant.

TABLE 2: Pentosidine, CML, and sRAGE levels in control subjects and patients with MetS.

\begin{tabular}{|c|c|c|c|}
\hline & $\begin{array}{c}\text { Control subjects } \\
n=80\end{array}$ & $\begin{array}{c}\text { Patients } \\
n=86\end{array}$ & $P$ \\
\hline Pentosidine ( $\mathrm{pmol} / \mathrm{mL})$ & $53.15(36.61-60.34)$ & $211.21(69.94-379.89)$ & $<0.001$ \\
\hline $\mathrm{CML}(\mathrm{pg} / \mathrm{mL})$ & $171.75(81.03-298.06)$ & $440.38(383.98-601.36)$ & $<0.001$ \\
\hline sRAGE $(\mathrm{pg} / \mathrm{mL})$ & $117.33(104.38-158.04)$ & $155(126.34-240.4)$ & 0.014 \\
\hline
\end{tabular}

Values are median (25\%-75\%) percentiles; CML: N (carboxymethyl) lysine; MetS: metabolic syndrome; sRAGE: soluble receptor for AGE.

TABLE 3: Treatment effect of metformin on pentosidine and CML levels in patients with MetS.

\begin{tabular}{lccc}
\hline & Treated patients & Untreated patients & $n=31$ \\
& $n=55$ & $P$ & 0.01 \\
Pentosidine $(\mathrm{pmol} / \mathrm{mL})$ & $140.72(62.07-249.51)$ & $372.45(132.55-556.68)$ & \\
CML $(\mathrm{pg} / \mathrm{mL})$ & $504.39(405.52-784.7)$ & $439.76(273.12-460.98)$ & 0.083 \\
\hline
\end{tabular}

Values are median (25\%-75\%) percentiles; CML: N (carboxymethyl) lysine.

control subjects. Patients showed an increased level of pentosidine, CML, and sRAGE compared to control subjects (211.21 (69.94-379.89) versus 53.15 (36.61-60.34) $\mathrm{pmol} / \mathrm{mL}$; 440.38 (383.98-601.36) versus 171.75 (81.03-298.06) pg/mL; 155 (126.34-240.4) versus 117.33 (104.38-158.04) pg/mL; $P<$ $0.001, P<0.001$, and $P=0.014$, resp.).
3.3. Treatment Effect of Metformin on Pentosidine, CML, and sRAGE Levels in Patients with MetS. As shown in Table 3, the plasma levels of pentosidine were significantly decreased between patients who received metformin and patients without this treatment: $140.72(62.07-249.51)$ versus $372.45(132.55-556.68) \mathrm{pmol} / \mathrm{mL}(P=0.01)$, respectively. 


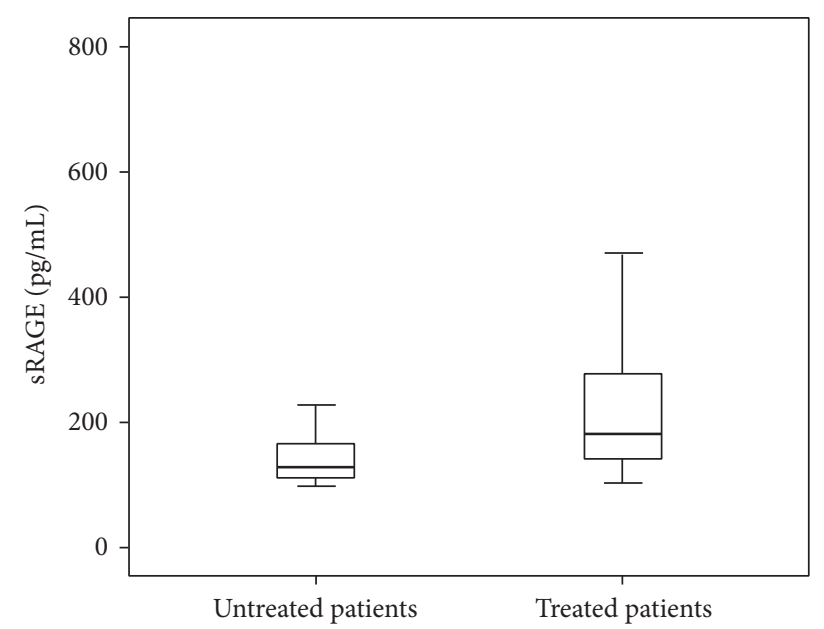

FIgURE 1: Box plots of plasma sRAGE levels in treated $(n=55)$ and untreated $(n=31)$ patients with metformin. The horizontal lines in each box represent (bottom to top) the 10th, 25th, 50th (median), 75th, and 90th percentiles. sRAGE: soluble receptor for AGE $(P<$ 0.001 ).

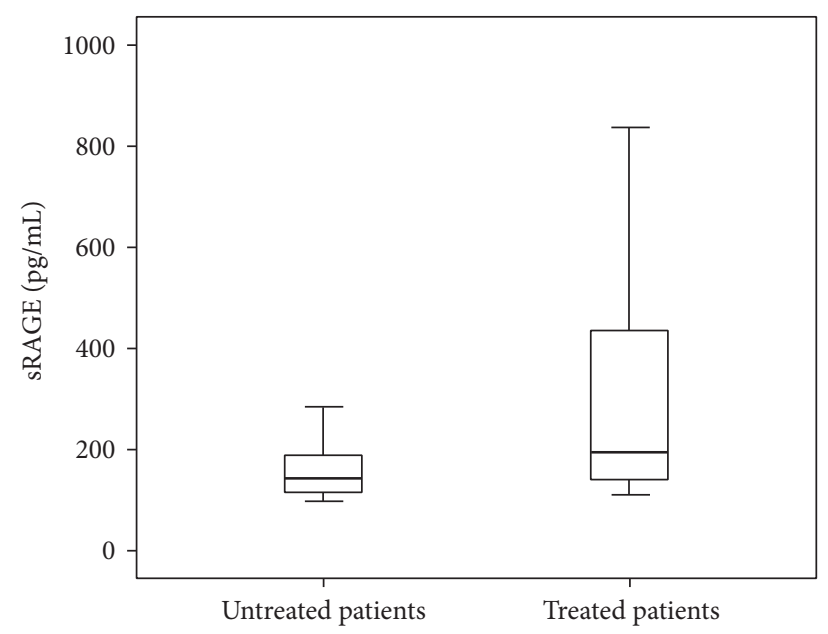

FIgUre 2: Box plots of plasma sRAGE levels in treated $(n=33)$ and untreated $(n=53)$ patients with ACE inhibitors $(P=0.002)$.

However, no significant difference between metformin effects on plasma CML levels had been shown. Plasma levels of sRAGE were significantly increased between patients who received metformin and patients without this treatment: $180.9(142.33-273.85)$ versus $131.84(111.75-226.98) \mathrm{pg} / \mathrm{mL}$ $(P<0.001)$, respectively. We cannot conclude that this increase is due to the effect of metformin because there is an increase in sRAGE plasma levels in patients who are treated with ACE inhibitors: 194.9 (138.59-468.59) versus 142.33 $(116.69-194.32) \mathrm{pg} / \mathrm{mL}$ in untreated patients $(P=0.002)$. This finding was illustrated in Figures 1 and 2. However, no significant difference between treated patients and untreated patients with other drugs on plasma pentosidine and CML levels had been shown.

3.4. Linear Regression and Multivariate Analyses. A linear regression analysis in patients with MetS showed negative and significant correlation between pentosidine levels and metformin treatment. sRAGE levels correlated positively with metformin treatment and/or ACE inhibitors treatment. However, no significant correlation between drugs treatments and CML levels had been shown (Table 4). To explain the levels variance of pentosidine and sRAGE in patients who received metformin, multiple linear regression model was formed. This model, which was applied to different drugs treatment (considered as independent variables) as well as pentosidine and sRAGE as the dependent variable, showed that these two biomarkers are not correlated positively to the treatment (Table 5).

\section{Discussion}

Predictors and identification of factors involved in the modulation of metabolic disorders of metabolic syndrome may be important in the prevention and the management of their complications. For this context, in the first part, the present study demonstrated new biomarkers such as plasma pentosidine, CML, and sRAGE levels, which are increased in patients with MetS. In the second part, our study evaluated the levels of these biomarkers in patients treated with drugs recognized by their antiglycation effect as metformin. Our results showed decreased pentosidine and increased sRAGE plasma levels in treated patients compared with untreated patients.

A number of clinical studies have reported that AGEs accumulate at a much higher rate in diabetic than in normal population [23-25]. Other studies have shown that circulating levels of AGEs are positively associated with inflammatory markers, endothelial dysfunction, insulin resistance, and vascular complications in diabetes [26-29]. Serum levels of pentosidine were found to be significantly higher in patients with diabetes than those without diabetes. Moreover, pentosidine levels were significantly higher in diabetic patients with cardiovascular disease than in those without cardiovascular disease $[30,31]$. Kerkeni et al. found that pentosidine is a biomarker for microvascular complications in type 2 diabetic patients and an independent determinant of the presence of hypertension and hyperlipidaemia [8]. Indicators of the degree of vascular damage, on cardiovascular disease, are dramatically amplified when diabetes is added to dyslipidemia, hypertension, and obesity. Our study showed that pentosidine levels were markedly increased in patients with MetS compared with our previous studies. However, no specific biomarkers have been studied to resolve the tsunami effect of metabolic syndrome, as a multifactorial disease. In addition to the interest effects of pentosidine, many studies have reported that CML serum levels are higher in diabetic patients compared to nondiabetic patients [3234]. Other results have showed a positive association between serum CML and vascular complication in diabetes [3537]. According to these results, our study showed increased plasma levels of CML in patients with MetS compared to control subjects.

The reason why AGEs are involved in the genesis of metabolic syndrome needs to be explained. At the molecular levels, AGEs including pentosidine and CML cause damage 
TABLE 4: Correlation between pentosidine, CML, sRAGE levels and drugs treatment in patients with MetS: linear regression analysis.

\begin{tabular}{|c|c|c|c|c|c|c|}
\hline \multirow{2}{*}{ Variables } & \multicolumn{2}{|c|}{ Pentosidine } & \multicolumn{2}{|c|}{ CML } & \multicolumn{2}{|c|}{ sRAGE } \\
\hline & $r$ & $P$ & $r$ & $P$ & $r$ & $P$ \\
\hline Metformin treatment & -0.311 & 0.009 & 0.177 & NS & 0.401 & $<0.001$ \\
\hline ACE inhibitors treatment & -0.054 & NS & 0.021 & NS & 0.352 & 0.002 \\
\hline Metformin + ACE inhibitors treatment & -0.071 & NS & 0.07 & NS & 0.408 & $<0.001$ \\
\hline
\end{tabular}

TABLE 5: Correlation between pentosidine, sRAGE, and drugs treatments in patients with MetS: multivariate regression analysis.

\begin{tabular}{lcccc}
\hline \multirow{2}{*}{ Variables } & \multicolumn{2}{c}{ Pentosidine } & \multicolumn{2}{c}{ sRAGE } \\
& $\beta$ & $P$ & $\beta$ & $P$ \\
\hline $\begin{array}{l}\text { Metformin } \\
\text { treatment }\end{array}$ & -0.246 & 0.099 & 0.045 & 0.73 \\
$\begin{array}{l}\text { ACE inhibitors } \\
\text { treatment }\end{array}$ & -0.299 & 0.182 & 0.08 & 0.685 \\
$\begin{array}{l}\text { Metformin + ACE } \\
\text { inhibitors treatment }\end{array}$ & -0.236 & 0.951 & 0.326 & 0.143 \\
\hline
\end{tabular}

ANOVA revealed a statistically not significant fit $(P=0.228)$.

A stepwise multivariate regression was performed.

by two principal mechanisms. First, structural modification of proteins perturbs their normal function. Second, AGEs are ligands for the receptor for AGEs (RAGE) causes intracellular oxidative stress and activation of nuclear factor NF$\mathrm{K} \beta$ who modulates genes transcription for various factors: endothelin-1, vascular endothelial growth factor (VEGF), transforming growth factor- $\beta$ (TGF- $\beta$ ), proinflammatory cytokines such as interleukins 1 and 6 , and tumor necrosis factor- $\alpha$ (TNF- $\alpha)[38,39]$. As a consequence, AGEs are related to wide variety of systemic pathologic conditions including ageing, hypertension, renal failure, diabetes, or cardiovascular complications [40].

A several metabolic components well established as risk factors for cardiovascular disease have also been shown to be associated with altered plasma sRAGE or esRAGE. Plasma esRAGE levels are decreased in patients with MetS and are inversely correlated with several components of MetS including body mass index, blood pressures, insulin resistance index, fasting plasma glucose, serum triglyceride, and lower HDL cholesterol levels [41]. Hudson et al. in the Northern Manhattan study demonstrated that lower sRAGE levels are not only associated with MetS but also lower in proportion to the number of metabolic components [42]. The findings regarding the soluble form of RAGE in diabetes are quite confusing. The same groups have found that plasma esRAGE and sRAGE levels are significantly lower in type 1 and type 2 diabetic patients than in nondiabetic controls $[43,44]$. Other groups found that plasma sRAGE levels increased in type 1 and type 2 diabetic patients [45-47]. This confusion in the plasma sRAGE levels may be due to the treatment effect. sRAGE levels are affected by drugs such as ACE inhibitors, and patients treated with this drugs have significantly higher levels of circulating sRAGE [48]. Our study demonstrated that plasma levels of sRAGE were significantly increased in treated patients with metformin compared to untreated patients but we cannot conclude that this increase is due to the effect of metformin because there is an increase in sRAGE plasma levels in patients treated with ACE inhibitors.

In addition to its action on glycemic control through the decrease in hepatic glucose production and increasing the sensitivity of peripheral cells to insulin, a number of studies have shown that metformin is beneficial in reducing diabetes associated vascular risk by inhibition of glycation process and the intermediate of AGEs such as MG $[49,50]$. The chemical structure of this drug is related to that of aminoguanidine which could be the highlight for inhibiting the formation of AGEs [51]. Diamanti-Kandarakis et al. have shown that plasma AGEs levels were reduced after 6 months of metformin administration in women with polycystic ovary syndrome [52]. Other studies have confirmed that metformin can reduce AGEs levels in lenses, kidneys, and nerves in diabetic animals [53]. According to these results, our study showed a decreased levels of pentosidine but not CML in patients who are treated with metformin. The mechanism by which metformin lowers plasma levels of pentosidine and not those of CML in the studied group of MetS is not clear; however, there is evidence that its effect could interfere with formation pathway. Pentosidine could be formed by the reaction of ribose, glucose, fructose, or ascorbate with lysine and arginine [54]. Particularly, CML could be formed by the reaction of glucose and other sugars and glyoxal with amino groups of arginine and lysine or by lipids peroxidation [55]. So, the rate of CML formation in patients can be dominated by the inhibition capacity of metformin.

\section{Conclusion}

Patients with metabolic syndrome showed increased levels of AGEs products such as pentosidine and CML. Metformin treatment effect showed a decreased level of pentosidine but not of CML. Therapeutic ways of AGEs development should be taken into account and further experimental and in vitro studies merit for advanced research.

\section{Abbreviations}

AGEs: Advanced glycation end products

ACE: Angiotensin converting enzyme

CML: Carboxymethyl-lysine

MetS: Metabolic syndrome

sRAGE: Soluble receptor for advanced glycation end products

esRAGE: Endogenous secretory RAGE

MG: Methylglyoxal

ELISA: Enzyme-linked immunosorbent assay. 


\section{Disclosure}

The funding organization(s) played no role in the study design; in the collection, analysis, and interpretation of data; in the writing of the report; or in the decision to submit the report for publication. All the authors have accepted responsibility for the entire content of this submitted manuscript and approved submission.

\section{Competing Interests}

The authors declare that they have no competing interests.

\section{Acknowledgments}

This work was supported by funds from the Biochemistry Laboratory, LR12ES05, Faculty of Medicine, University of Monastir.

\section{References}

[1] S. O’Neill and L. O’Driscoll, "Metabolic syndrome: A closer look at the growing epidemic and its associated pathologies," Obesity Reviews, vol. 16, no. 1, pp. 1-12, 2015.

[2] D. G. Dyer, J. A. Blackledge, S. R. Thorpet, and J. W. Baynes, "Formation of pentosidine during nonenzymatic browning of proteins by glucose. Identification of glucose and other carbohydrates as possible precursors of pentosidine in vivo," The Journal of Biological Chemistry, vol. 266, no. 18, pp. 11654-11660, 1991.

[3] M. Takeuchi and S.-I. Yamagishi, "Involvement of toxic AGEs (TAGE) in the pathogenesis of diabetic vascular complications and Alzheimer's disease," Journal of Alzheimer's Disease, vol. 16, no. 4, pp. 845-858, 2009.

[4] K. Yang, D. Qiang, S. Delaney, R. Mehta, W. R. Bruce, and P. J. O'Brien, "Differences in glyoxal and methylglyoxal metabolism determine cellular susceptibility to protein carbonylation and cytotoxicity," Chemico-Biological Interactions, vol. 191, no. 1-3, pp. 322-329, 2011.

[5] F. J. Tessier and I. Birlouez-Aragon, "Health effects of dietary Maillard reaction products: the results of ICARE and other studies," Amino Acids, vol. 42, no. 4, pp. 1119-1131, 2012.

[6] A. A. Ghanem, A. Elewa, and L. F. Arafa, "Pentosidine and Ncarboxymethyl-lysine: biomarkers for type 2 diabetic retinopathy," European Journal of Ophthalmology, vol. 21, no. 1, pp. 4854, 2011.

[7] M. Kerkeni, A. Saïdi, H. Bouzidi, S. B. Yahya, and M. Hammami, "Elevated serum levels of AGEs, sRAGE, and pentosidine in Tunisian patients with severity of diabetic retinopathy," Microvascular Research, vol. 84, no. 3, pp. 378-383, 2012.

[8] M. Kerkeni, A. Saïdi, H. Bouzidi, A. Letaief, S. Ben Yahia, and M. Hammami, "Pentosidine as a biomarker for microvascular complications in type 2 diabetic patients," Diabetes and Vascular Disease Research, vol. 10, no. 3, pp. 239-245, 2013.

[9] M. Kerkeni, I. S. Weiss, S. Jaisson et al., "Increased serum concentrations of pentosidine are related to presence and severity of coronary artery disease," Thrombosis Research, vol. 134, no. 3, pp. 633-638, 2014.

[10] J. S. Nyman, A. Roy, R. L. Acuna et al., "Age-related effect on the concentration of collagen crosslinks in human osteonal and interstitial bone tissue," Bone, vol. 39, no. 6, pp. 1210-1217, 2006.
[11] J. M. Haus, J. A. Carrithers, S. W. Trappe, and T. A. Trappe, "Collagen, cross-linking, and advanced glycation end products in aging human skeletal muscle," Journal of Applied Physiology, vol. 103, no. 6, pp. 2068-2076, 2007.

[12] C. Ott, K. Jacobs, E. Haucke, A. Navarrete Santos, T. Grune, and A. Simm, "Role of advanced glycation end products in cellular signaling," Redox Biology, vol. 2, no. 1, pp. 411-429, 2014.

[13] A. Z. Kalea, A. M. Schmidt, and B. I. Hudson, "RAGE: a novel biological and genetic marker for vascular disease," Clinical Science, vol. 116, no. 8, pp. 621-637, 2009.

[14] K. Kierdorf and G. Fritz, "RAGE regulation and signaling in inflammation and beyond," Journal of Leukocyte Biology, vol. 94, no. 1, pp. 55-68, 2013.

[15] S.-I. Yamagishi, T. Matsui, and K. Fukami, "Role of receptor for advanced glycation end products (RAGE) and its ligands in cancer risk," Rejuvenation Research, vol. 18, no. 1, pp. 48-56, 2015.

[16] Z. Cai, N. Liu, C. Wang et al., "Role of RAGE in Alzheimer's disease," Cellular and Molecular Neurobiology, vol. 36, no. 4, pp. 483-495, 2016.

[17] M. Monden, H. Koyama, Y. Otsuka et al., "Receptor for advanced glycation end products regulates adipocyte hypertrophy and insulin sensitivity in mice: involvement of toll-like receptor 2," Diabetes, vol. 62, no. 2, pp. 478-489, 2013.

[18] L. Zhang, M. Bukulin, E. Kojro et al., "Receptor for advanced glycation end products is subjected to protein ectodomain shedding by metalloproteinases," Journal of Biological Chemistry, vol. 283, no. 51, pp. 35507-35516, 2008.

[19] F. Piarulli, A. Lapolla, E. Ragazzi et al., "Role of endogenous secretory RAGE (esRAGE) in defending against plaque formation induced by oxidative stress in type 2 diabetic patients," Atherosclerosis, vol. 226, no. 1, pp. 252-257, 2013.

[20] D. Geroldi, C. Falcone, and E. Emanuele, "Soluble receptor for advanced glycation end products: from disease marker to potential therapeutic target," Current Medicinal Chemistry, vol. 13, no. 17, pp. 1971-1978, 2006.

[21] S. F. Yan, R. Ramasamy, and A. M. Schmidt, "Soluble RAGE: therapy and biomarker in unraveling the RAGE axis in chronic disease and aging," Biochemical Pharmacology, vol. 79, no. 10, pp. 1379-1386, 2010.

[22] K. G. M. M. Alberti, R. H. Eckel, S. M. Grundy et al., "Harmonizing the metabolic syndrome: a joint interim statement of the International Diabetes Federation Task Force on Epidemiology and Prevention; National Heart, Lung, and Blood Institute; American Heart Association; World Heart Federation; International Atherosclerosis Society; and International Association for the Study of Obesity," Circulation, vol. 120, no. 16, pp. 16401645,2009

[23] B. K. Kilhovd, T. J. Berg, K. I. Birkeland, P. Thorsby, and K. F. Hanssen, "Serum levels of advanced glycation end products are increased in patients with type 2 diabetes and coronary heart disease," Diabetes Care, vol. 22, no. 9, pp. 1543-1548, 1999.

[24] M. C. Thomas, M. Woodward, B. Neal et al., "Relationship between levels of advanced glycation end products and their soluble receptor and adverse outcomes in adults with type 2 diabetes," Diabetes Care, vol. 38, no. 10, pp. 1891-1897, 2015.

[25] N. M. Hanssen, J. W. Beulens, S. van Dieren et al., "Plasma advanced glycation end products are associated with incident cardiovascular events in individuals with type 2 diabetes: a casecohort study with a median follow-up of 10 years (EPIC-NL)," Diabetes, vol. 64, no. 1, pp. 257-265, 2015. 
[26] K. C. B. Tan, S. W. M. Shiu, Y. Wong, and X. Tam, "Serum advanced glycation end products (AGEs) are associated with insulin resistance," Diabetes/Metabolism Research and Reviews, vol. 27, no. 5, pp. 488-492, 2011.

[27] K. Nakamura, S.-I. Yamagishi, H. Adachi et al., "Circulating advanced glycation end products (AGEs) and soluble form of receptor for AGEs (sRAGE) are independent determinants of serum monocyte chemoattractant protein-1 (MCP-1) levels in patients with type 2 diabetes," Diabetes/Metabolism Research and Reviews, vol. 24, no. 2, pp. 109-114, 2008.

[28] K. C. B. Tan, W.-S. Chow, V. H. G. Ai, C. Metz, R. Bucala, and K. S. L. Lam, "Advanced glycation end products and endothelial dysfunction in type 2 diabetes," Diabetes Care, vol. 25, no. 6, pp. 1055-1059, 2002.

[29] S.-I. Yamagishi, K. Fukami, and T. Matsui, "Evaluation of tissue accumulation levels of advanced glycation end products by skin autofluorescence: a novel marker of vascular complications in high-risk patients for cardiovascular disease," International Journal of Cardiology, vol. 185, pp. 263-268, 2015.

[30] S. Sugiyama, T. Miyata, Y. Ueda et al., "Plasma levels of pentosidine in diabetic patients: an advanced glycation end product," Journal of the American Society of Nephrology, vol. 9, no. 9, pp. 1681-1688, 1998.

[31] N. Yoshida, K.-I. Okumura, and Y. Aso, "High serum pentosidine concentrations are associated with increased arterial stiffness and thickness in patients with type 2 diabetes," Metabolism: Clinical and Experimental, vol. 54, no. 3, pp. 345-350, 2005.

[32] Z. Wagner, I. Wittmann, I. Mazak et al., "Ne-(Carboxymethyl)lysine levels in patients with type 2 diabetes: role of renal function," American Journal of Kidney Diseases, vol. 38, no. 4, pp. 785-791, 2001.

[33] V. C. Luft, B. B. Duncan, M. I. Schmidt et al., "Carboxymethyl lysine, an advanced glycation end product, and incident diabetes: a case-cohort analysis of the ARIC Study," Diabetic Medicine, vol. 33, no. 10, pp. 1392-1398, 2016.

[34] A. F. M. L. Lieuw, V. W. M. van Hinsbergh, T. Teerlink et al., "Increased levels of $N^{\varepsilon}$-(carboxymethyl)lysine and $N^{\varepsilon}$ (carboxyethyl)lysine in type 1 diabetic patients with impaired renal function: correlation with markers of endothelial dysfunction," Nephrology Dialysis Transplantation, vol. 19, no. 3, pp. 631636, 2004.

[35] M. P. Wautier, P. Massin, P. J. Guillausseau et al., "N(carboxymethyl)lysine as a biomarker for microvascular complications in type 2 diabetic patients," Diabetes and Metabolism, vol. 29, no. 1, pp. 44-52, 2003.

[36] N. Mishra, S. Saxena, R. K. Shukla et al., "Association of serum N $\varepsilon$-Carboxy methyl lysine with severity of diabetic retinopathy," Journal of Diabetes and Its Complications, vol. 30, no. 3, pp. 511517, 2016.

[37] S. Choudhuri, D. Dutta, A. Sen et al., "Role of N-epsiloncarboxy methyl lysine, advanced glycation end products and reactive oxygen species for the development of nonproliferative and proliferative retinopathy in type 2 diabetes mellitus," Molecular Vision, vol. 19, pp. 100-113, 2013.

[38] D. P. Barlovic, A. Soro-Paavonen, and K. A. M. Jandeleit-Dahm, "RAGE biology, atherosclerosis and diabetes," Clinical Science, vol. 121, no. 2, pp. 43-55, 2011.

[39] G. Basta, A. M. Schmidt, and R. De Caterina, "Advanced glycation end products and vascular inflammation: implications for accelerated atherosclerosis in diabetes," Cardiovascular Research, vol. 63, no. 4, pp. 582-592, 2004.
[40] Z. Hegab, S. Gibbons, L. Neyses, and M. A. Mamas, "Role of advanced glycation end products in cardiovascular disease," World Journal of Cardiology, vol. 4, no. 4, pp. 90-102, 2012.

[41] H. Koyama, T. Shoji, H. Yokoyama et al., "Plasma level of endogenous secretory RAGE is associated with components of the metabolic syndrome and atherosclerosis," Arteriosclerosis, Thrombosis, and Vascular Biology, vol. 25, no. 12, pp. 2587-2593, 2005.

[42] B. I. Hudson, C. Dong, H. Gardener et al., "Serum levels of soluble receptor for advanced glycation end-products and metabolic syndrome: the Northern Manhattan Study," Metabolism: Clinical and Experimental, vol. 63, no. 9, pp. 1125-1130, 2014.

[43] N. Katakami, M. Matsuhisa, H. Kaneto et al., "Decreased endogenous secretory advanced glycation end product receptor in type 1 diabetic patients: its possible association with diabetic vascular complications," Diabetes Care, vol. 28, no. 11, pp. 27162721, 2005.

[44] N. Grossin, M.-P. Wautier, T. Meas, P.-J. Guillausseau, P. Massin, and J.-L. Wautier, "Severity of diabetic microvascular complications is associated with a low soluble RAGE level," Diabetes and Metabolism, vol. 34, no. 4, pp. 392-395, 2008.

[45] M. Challier, S. Jacqueminet, O. Benabdesselam, A. Grimaldi, and J.-L. Beaudeux, "Increased serum concentrations of soluble receptor for advanced glycation endproducts in patients with type 1 diabetes," Clinical Chemistry, vol. 51, no. 9, pp. 1749-1750, 2005.

[46] K. C. Tan, S. W. Shiu, W. S. Chow, L. Leng, R. Bucala, and D. J. Betteridge, "Association between serum levels of soluble receptor for advanced glycation end products and circulating advanced glycation end products in type 2 diabetes," Diabetologia, vol. 49, no. 11, pp. 2756-2762, 2006.

[47] K. Nakamura, S.-I. Yamagishi, H. Adachi et al., "Elevation of soluble form of receptor for advanced glycation end products (sRAGE) in diabetic subjects with coronary artery disease," Diabetes/Metabolism Research and Reviews, vol. 23, no. 5, pp. 368-371, 2007.

[48] J. M. Forbes, S. R. Thorpe, V. Thallas-Bonke et al., "Modulation of soluble receptor for advanced glycation end products by angiotensin-converting enzyme-1 inhibition in diabetic nephropathy," Journal of the American Society of Nephrology, vol. 16, no. 8, pp. 2363-2372, 2005.

[49] P. J. Beisswenger, S. K. Howell, A. D. Touchette, S. Lal, and B. S. Szwergold, "Metformin reduces systemic methylglyoxal levels in type 2 diabetes," Diabetes, vol. 48, no. 1, pp. 198-202, 1999.

[50] S. Ahmad, U. Shahab, M. H. Baig et al., "Inhibitory effect of metformin and pyridoxamine in the formation of early, intermediate and advanced glycation end-products," PLOS ONE, vol. 8, no. 9, Article ID e72128, 2013.

[51] J. Peyroux and M. Sternberg, "Advanced glycation endproducts (AGEs): pharmacological inhibition in diabetes," Pathologie Biologie, vol. 54, no. 7, pp. 405-419, 2006.

[52] E. Diamanti-Kandarakis, K. Alexandraki, C. Piperi et al., "Effect of metformin administration on plasma advanced glycation end product levels in women with polycystic ovary syndrome," Metabolism: Clinical and Experimental, vol. 56, no. 1, pp. 129134, 2007.

[53] Y. Tanaka, H. Uchino, T. Shimizu et al., "Effect of metformin on advanced glycation endproduct formation and peripheral nerve function in streptozotocin-induced diabetic rats," European Journal of Pharmacology, vol. 376, no. 1-2, pp. 17-22, 1999. 
[54] S. K. Grandhee and V. M. Monnier, "Mechanism of formation of the maillard protein cross-link pentosidine: glucose, fructose, and ascorbate as pentosidine precursors," The Journal of Biological Chemistry, vol. 266, no. 18, pp. 11649-11653, 1991.

[55] G. Vistoli, D. De Maddis, A. Cipak, N. Zarkovic, M. Carini, and G. Aldini, "Advanced glycoxidation and lipoxidation end products (AGEs and ALEs): an overview of their mechanisms of formation," Free Radical Research, vol. 47, supplement 1, pp. 3-27, 2013. 


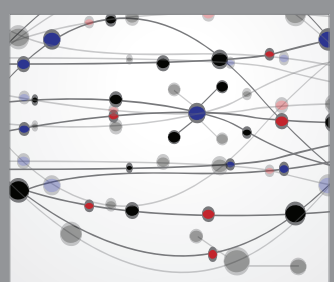

The Scientific World Journal
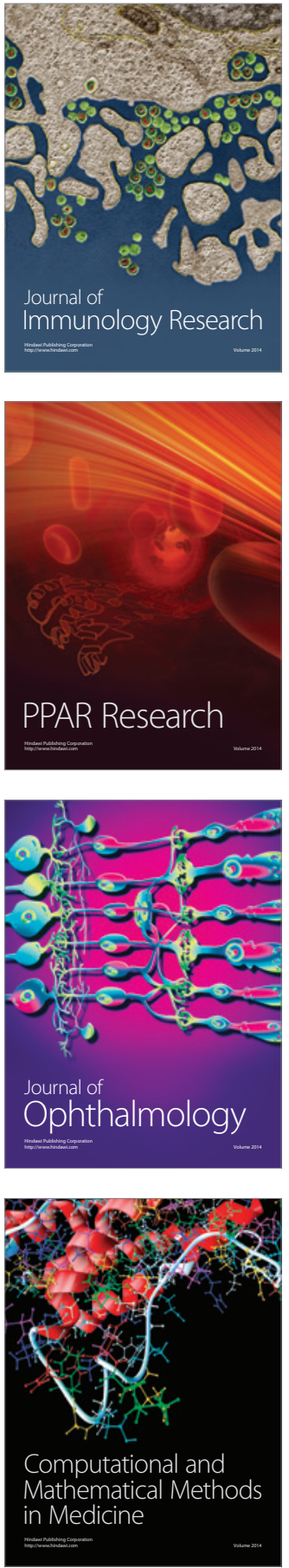

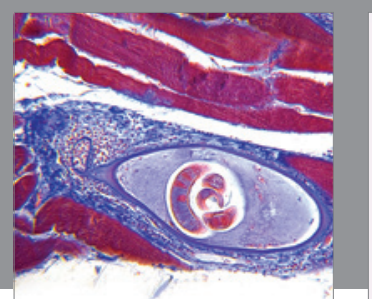

Gastroenterology Research and Practice

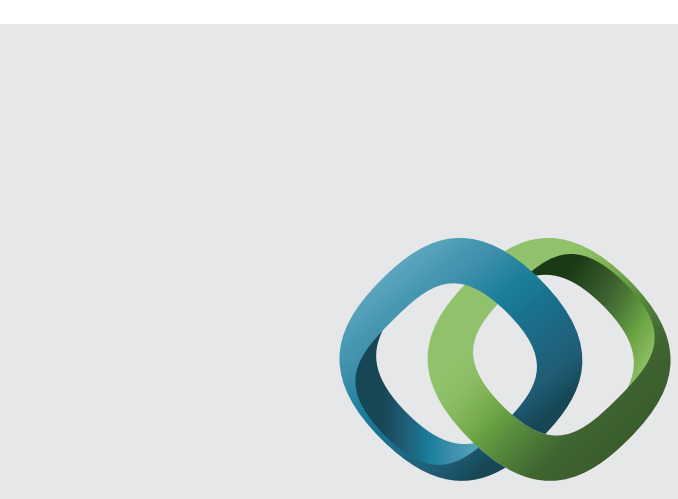

\section{Hindawi}

Submit your manuscripts at

http://www.hindawi.com
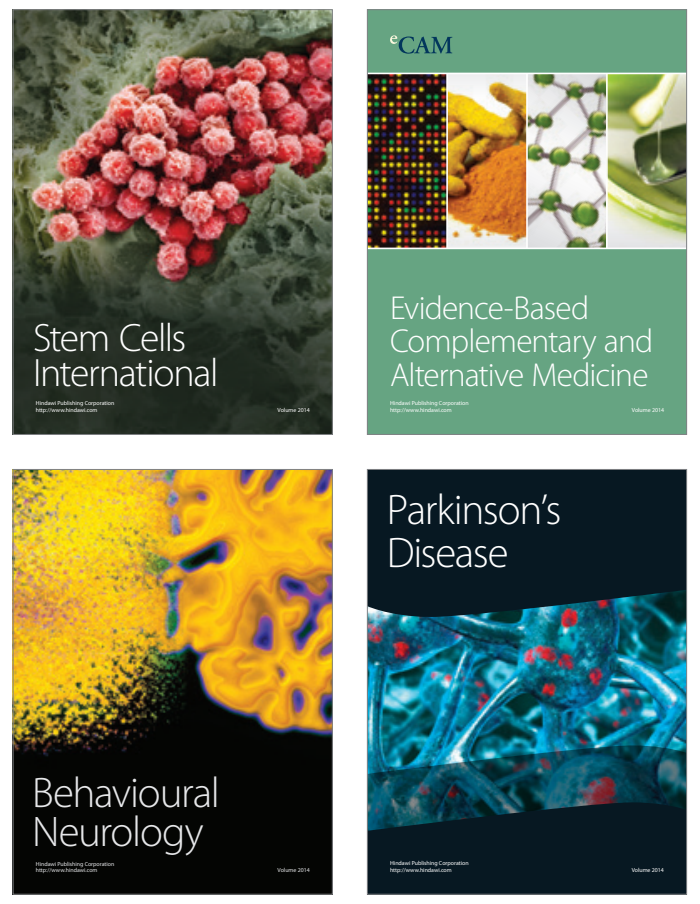
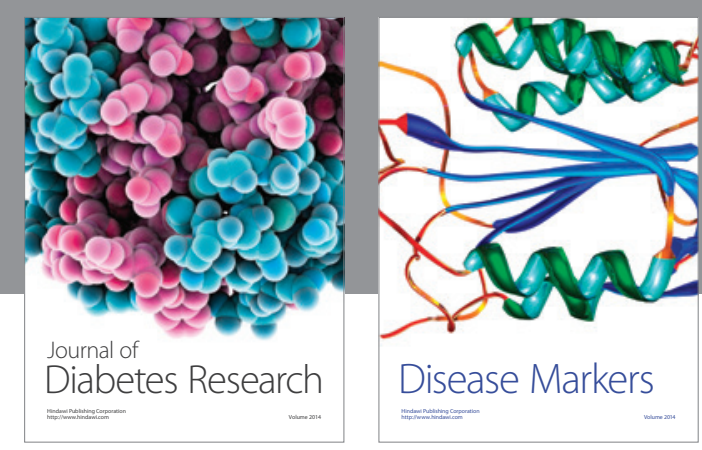

Disease Markers
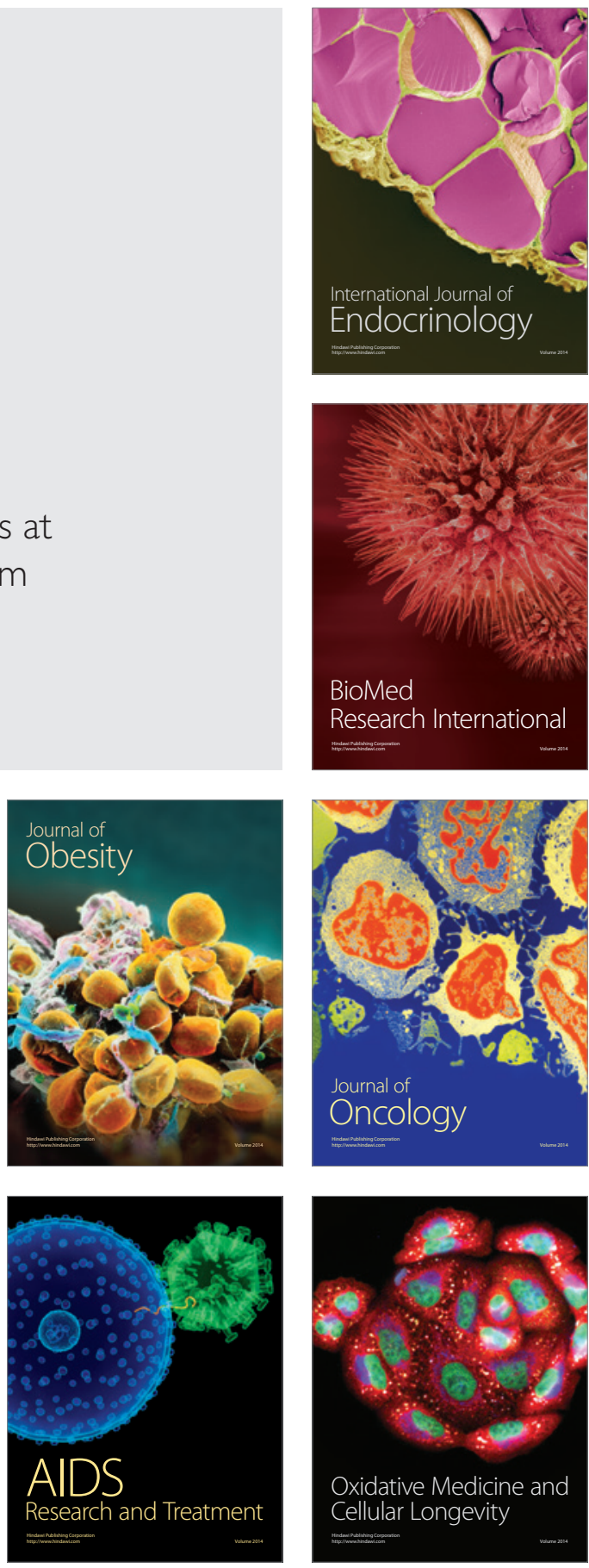